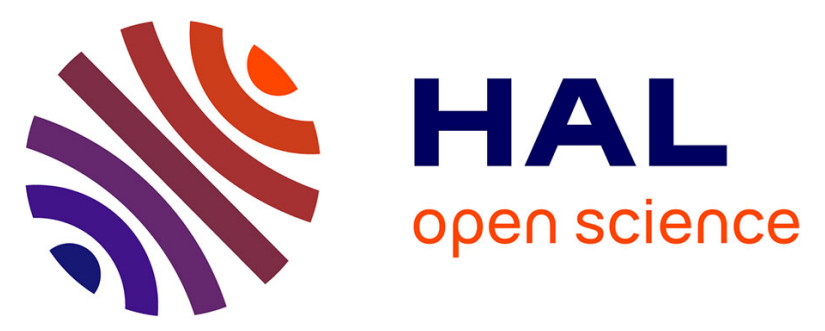

\title{
Solid-state NMR chemical-shift perturbations indicate domain reorientation of the DnaG primase in the primosome of Helicobacter pylori.
}

Carole Gardiennet, Thomas Wiegand, Alexandre Bazin, Riccardo Cadalbert, Britta Kunert, Denis Lacabanne, Irina Gutsche, Laurent Terradot, Beat H Meier, Anja Böckmann

\section{To cite this version:}

Carole Gardiennet, Thomas Wiegand, Alexandre Bazin, Riccardo Cadalbert, Britta Kunert, et al.. Solid-state NMR chemical-shift perturbations indicate domain reorientation of the DnaG primase in the primosome of Helicobacter pylori.. Journal of Biomolecular NMR, 2016, 64 (3), pp.189-95. 10.1007/s10858-016-0018-0 . hal-01443306

\section{HAL Id: hal-01443306 https://hal.univ-grenoble-alpes.fr/hal-01443306}

Submitted on 24 Nov 2020

HAL is a multi-disciplinary open access archive for the deposit and dissemination of scientific research documents, whether they are published or not. The documents may come from teaching and research institutions in France or abroad, or from public or private research centers.
L'archive ouverte pluridisciplinaire HAL, est destinée au dépôt et à la diffusion de documents scientifiques de niveau recherche, publiés ou non, émanant des établissements d'enseignement et de recherche français ou étrangers, des laboratoires publics ou privés. 


\section{$\boldsymbol{G H}$ zürich}

\section{Research Collection}

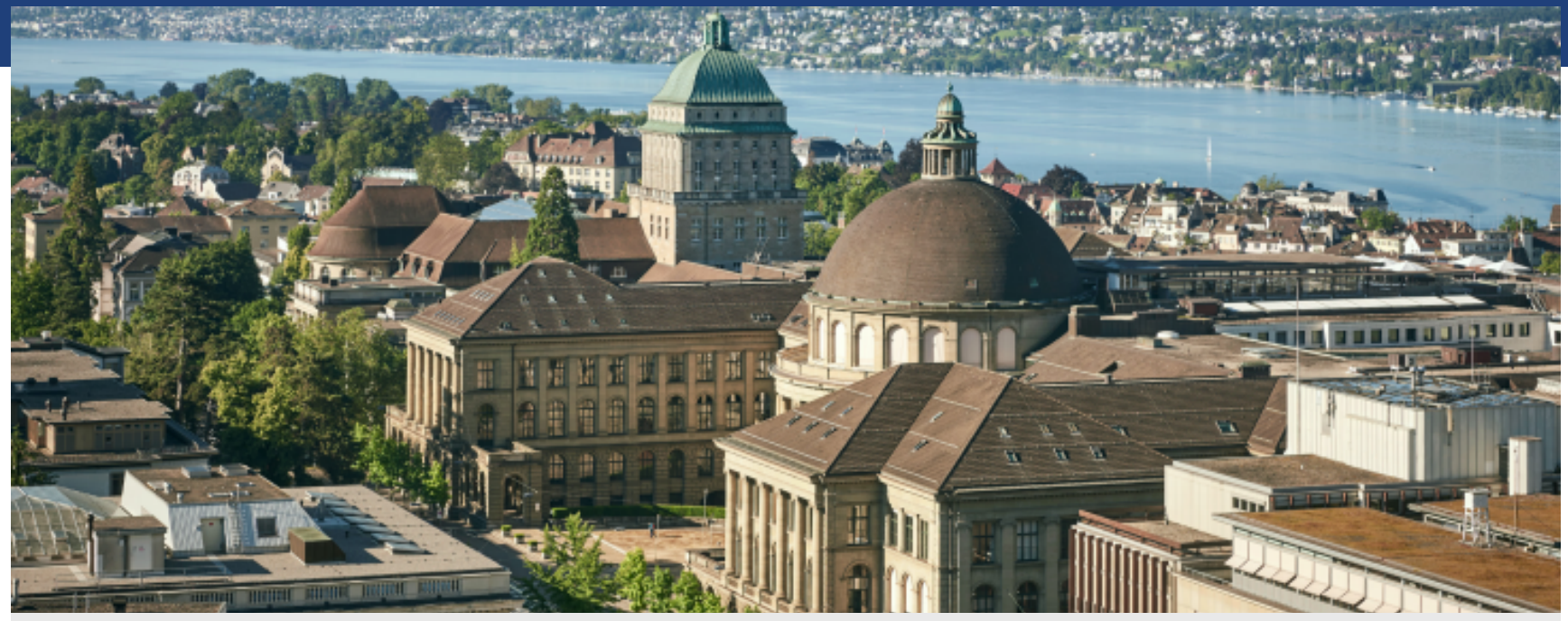

Journal Article

\section{Solid-state NMR chemical-shift perturbations indicate domain reorientation of the DnaG primase in the primosome of Helicobacter pylori}

\section{Author(s):}

Gardiennet, Carole; Wiegand, Thomas; Bazin, Alexandre; Cadalbert, Riccardo; Kunert, Britta; Lacabanne, Denis; Gutsche, Irina; Terradot, Laurent; Meier, Beat H.; Böckmann, Anja

\section{Publication Date:}

2016-03

\section{Permanent Link:}

https://doi.org/10.3929/ethz-b-000114271 $\rightarrow$

Originally published in:

Journal of Biomolecular NMR 64(3), http://doi.org/10.1007/s10858-016-0018-0 $\rightarrow$

Rights / License:

In Copyright - Non-Commercial Use Permitted $\rightarrow$ 


\title{
Solid-state NMR chemical-shift perturbations indicate domain
}

\section{reorientation of the DnaG primase in the primosome of Helicobacter pylori}

Carole Gardiennet ${ }^{\text {ał⿱ }}$, Thomas Wiegand ${ }^{\mathrm{b} \ddagger}$, Alexandre Bazin ${ }^{\mathrm{a}}$, Riccardo Cadalbert ${ }^{\mathrm{b}}$, Britta

Kunert $^{\mathrm{a}}$, Denis Lacabanne ${ }^{\mathrm{a}}$, Irina Gutsche ${ }^{\mathrm{c}}$, Laurent Terradot ${ }^{\mathrm{a}^{*}}$, Beat H. Meier ${ }^{\mathrm{b}^{*}}$, and Anja

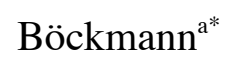

${ }^{a}$ Molecular Microbiology and Structural Biochemistry, Labex Ecofect, UMR 5086 CNRS, Université de Lyon 1, 7 passage du Vercors, 69007 Lyon, France

${ }^{b}$ Physical Chemistry, ETH Zurich, Vladimir-Prelog-Weg 2, 8093 Zurich (Switzerland)

${ }^{c}$ Université Grenoble Alpes, Institut de Biologie Structurale (IBS), CNRS, IBS; CEA, IBS, F38044 Grenoble, France

"present address : CNRS, Université de Lorraine, CRM2, UMR 7036, F-54506 Vandoeuvrelès-Nancy, France

*these authors contributed equally

corresponding authors: a.bockmann@ibcp.fr, beme@ethz.ch,l.terradot@ibcp.fr

\begin{abstract}
We here investigate the interactions between the DnaB helicase and the C-terminal domain (CTD) of the corresponding DnaG primase of Helicobacter pylori using solid-state NMR. The difficult crystallization of this $387 \mathrm{kDa}$ complex, where the two proteins interact in a six to three ratio, is circumvented by simple co-sedimentation of the two proteins directly into the MAS-NMR rotor. While the amount of information that can be extracted from such a large protein is still limited, we can assign a number of amino-acid residues experiencing significant chemical-shift perturbations upon helicase-primase complex formation. The location of these residues is used as a guide to model the interaction interface between the two proteins in the complex. Chemical-shift perturbation also reveals changes at the interaction interfaces of the hexameric $H p D n a B$ assembly on $H p D$ DaG binding. A structural model of the complex that explains the experimental findings is obtained.
\end{abstract}

\section{Introduction}


The DnaB helicase and the DnaG primase are proteins acting at the heart of the replication machinery which function is to produce identical copies of the genomic DNA of bacteria (Corn and Berger 2006). In order to do so, the DNA double helix is unwound by the DnaB helicase in the 5'-3' direction. DnaB also recruits the DnaG primase, which synthesizes RNA primers to be used as template by the DNA polymerase. The DnaB helicases generally function as hexamers, and the $\mathrm{N}$-terminal domain displays trimers of dimers arranged in a head-to-tail manner. Structures from DnaB domains of different organisms (Kashav et al. 2009; Stelter et al. 2012) and the interacting DnaG C-terminal domain (CTD), (Oakley et al. 2005; Abdul Rehman et al. 2013) as well as a complex of DnaB/DnaG-CTD from Bacillus stearothermophilus (Bst) (Bailey et al. 2007) are available.

The structures of different DnaG-CTDs reveal a fold very similar to the N-terminal domain of the DnaB helicase, consisting of a globular domain and a helical hairpin region (Oakley et al. 2005; Syson et al. 2005; Su et al. 2006; Abdul Rehman et al. 2013). The DnaG-CTD helical hairpin has been described to adopt multiple orientations relative to the globular domain in the crystal structures of isolated DnaG-CTDs from different organisms, and is thus believed to have a flexible orientation with respect to the globular domain. This has also been revealed by NMR studies, which showed that the linker region between the two domains is flexible and dynamic (Oakley et al. 2005).

Helicobacter pylori is a common pathogen and can be at the origin of various diseases, from chronic gastritis to gastric cancers (Parsonnet 1995). In H. pylori, DnaG (HpDnaG) and DnaB (HpDnaB) show in vitro moderate interactions when compared to other organisms as E. coli and B. stearothermophilus (Abdul Rehman et al. 2013), which may be a reason why crystallization of the complex proved difficult. For solid-state NMR studies, sedimentation of proteins has recently emerged as a valuable sample-preparation venue and enables the study of large soluble protein assemblies (Mainz et al. 2009; Bertini et al. 2011; Gardiennet et al. 
2012). This technique has been shown to yield high-resolution NMR spectra for several preparations, including of $H p D n a B$ (Gardiennet et al. 2012). We here use this approach to study a complex formed by two different interacting proteins, in which the large molecular weight of one protein multimer allows to sediment the complex it forms with a smaller interacting protein. The analysis of the chemical-shift changes of $H p D n a B$ allows to identify the interaction interfaces in the $H p D n a B / H p D n a G-C T D$ complex, and we devise a model in which the hairpin in the DnaG-CTD of the primase undergoes a conformational change with respect to its X-ray structure in isolation (Abdul Rehman et al. 2013) in order to comply with the observed chemical-shift perturbations.

$H p \mathrm{DnaB}$, in the presence of $H p \mathrm{DnaG}-\mathrm{CTD}$, forms a protein complex. In order to evaluate whether the two proteins cosediment, we analyzed the pellet and supernatant fractions after ultracentrifugation of a test sample (Fig. S1). We recorded $2 \mathrm{D}{ }^{13} \mathrm{C}-{ }^{13} \mathrm{C}$ solid-state NMR DARR spectra of the sedimented HpDnaB full-length protein (Gardiennet et al. 2012), as well as of the co-sedimented $H p D n a B / H p D n a G-C T D$ sample. Spectra of the isolated N-terminal domain (NTD) of $H p D n a B$, which has been sequentially assigned (Wiegand et al. 2015), as well as the C-terminal domain (CTD) of $H p D$ naB (both in crystalline form), served as a reference in this work.

\section{Results}

An overlay of the DARR spectra from sedimented ${ }^{13} \mathrm{C},{ }^{15} \mathrm{~N}$ labeled $H p D n a B$ and the cosedimented protein complex between ${ }^{13} \mathrm{C},{ }^{15} \mathrm{~N}$ labeled $H p D$ naB and $H p$ DnaG-CTD (at natural isotopic abundance) is shown in Fig. 1. The quality of both spectra is comparable, with linewidths (full-width at half maximum) of isolated signals of about $0.5 \mathrm{ppm}$. Spectra of sedimented $H p \mathrm{DnaB}$ share common features when $H p \mathrm{DnaB}$ is alone or in the presence of $H p$ DnaG-CTD, which indicates, not surprisingly, that the overall conformation of $H p D$ naB is 
conserved in the co-sediment. A closer look at the superposition of the two spectra, however, allows to identify clear spectral modifications due to the presence of $H p$ DnaG-CTD (which itself is not visible as it is not isotopically labeled). This indicates that the co-sedimented sample represents a well-defined protein complex and that DnaB undergoes local conformational changes when interacting with DnaG.

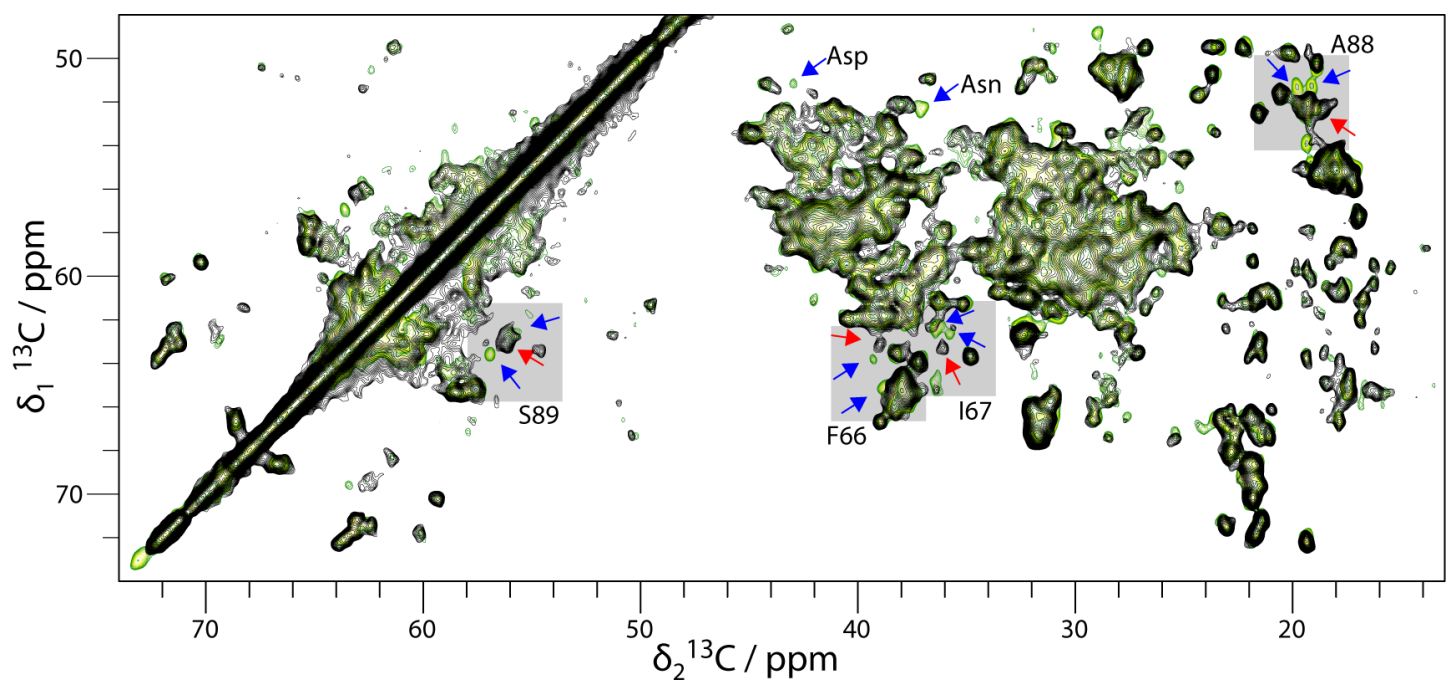

Figure 1. Superposition of the aliphatic regions of $20 \mathrm{~ms}$ DARR spectra recorded on the $H p \mathrm{DnaB} / H p \mathrm{DnaG}-\mathrm{CTD}$ co-sediment (green) and the $H p \mathrm{DnaB}$ sediment (black). Red arrows indicate signals which "disappear" in the spectra of the sedimented $H p D$ DaB, and blue arrows those which are "newly" observed in the $H p D$ naB/HpDnaG-CTD cosediment and which will be further discussed in the text. Extracts corresponding to the grey regions are shown in Fig. 2 . Individual spectra are given in the Supporting Information (Fig. S2), as well as the full aliphatic region including new signals in the isoleucine side-chain region (Fig. S3).

The most important chemical shift changes concern resonances that appear only in one of the two spectra. These signals can be classified into two categories: resonances present in $H p D$ naB but no longer observed in the protein complex (red arrows in Fig.1), and resonances 
which appear only in the spectra of the protein complex (blue arrows in Fig.1, see also Fig. S3). Most probably, these resonances belong to the same residues but are shifted, as a consequence of the complex formation inducing a considerable chemical-shift perturbation (CSP). For further resonances, small changes in chemical shift were observed. As they lie either within or very close to the experimental error which we estimate to about $0.3 \mathrm{ppm}$, we will not further discuss these. Other differences between the two spectra are explained by slight intensity changes in the corresponding resonances, e.g. resonances that drop below the lowest contour level in one of the spectra but can still identified in both spectra upon detailed inspection.

Residues that disappear from NMR spectra are either no longer observed due to dynamical or statical disorder, or shift so far that they can not be easily paired with the original resonance. The latter is in the current context more likely since, considering the appearance of narrow signals on interaction, spectral evidences for increasing disorder or dynamics on complex formation are not detected in the NMR spectra. Chemical shifts are a highly sensitive probe for conformational changes induced by interactions with partner molecules (for a recent review, see (Williamson 2013)). While solution-state protein-ligand interactions are often found to be in the fast exchange limit for NMR, with continuous titration curves for the CSP, the protein complexes observed here are not in equilibrium with the unbound $H p D$ DaB and we observed only disappearing signals from the $H p \mathrm{DnaB}$ and new signals for the complex arising.

For full-length $H p D n a B$, sequence specific assignments of the peaks of interest can be obtained by comparing the spectra of $H p D n a B$ to spectra recorded on its isolated N-terminal and C-terminal domains. If the peaks overlap within $0.3 \mathrm{ppm}$ with signals which were assigned in the N-terminal domain (Wiegand et al. 2015), and if no resonance from the Cterminal domain is found in vicinity, the assignment from the isolated $\mathrm{N}$-terminal domain can be taken over. This is illustrated in Fig. 2 where an overlay, as well as the individual spectra, 
are displayed of extracts from $H p$ DnaB-NTD (Wiegand et al. 2015), $H p$ DnaB, and also the $H p \mathrm{DnaB} / \mathrm{HpDnaG-CTD}$ protein complex. Four of the disappearing peaks could be assigned this way, F66, I67, A88 and S89. One more Ala peak is disappearing, but it could not be assigned, since it does not correspond to a signal observed in the isolated $\mathrm{N}$ - or C-terminal domains.

Correspondingly, several peaks are only present in the $H p D n a B / H p D n a G-C T D$ spectra, which are highlighted by blue arrows in Figs. 1 and S3. Tentative assignments of several appearing resonances can be proposed by pairing them with the most nearby disappearing resonances (grey boxes in Figs. 1 and S3). For F66, I67, A88 and S89, actually two newly appearing peaks are detected in close vicinity. This is shown in the right-most column of Fig. 2, where in the $H p \mathrm{DnaB} / \mathrm{HpDnaG-CTD}$ spectra these appearing signals are labeled with red crosses. The CSP as identified under these assumptions are quantified in Table S1. 


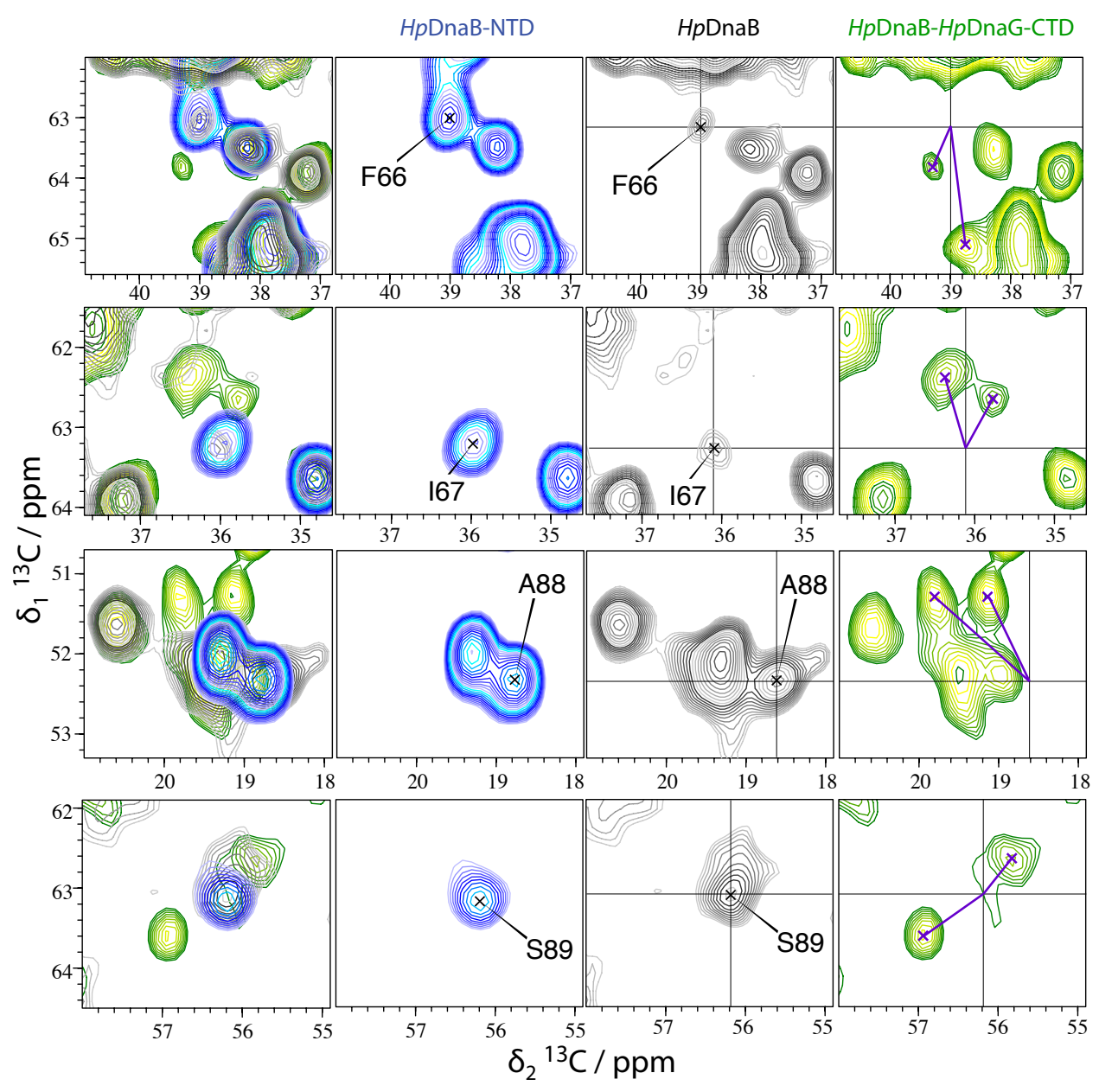

Figure 2: $2 \mathrm{D}$ extracts of $20 \mathrm{~ms}$ DARR spectra recorded on the $H p$ DnaB-NTD (in blue), $H p \mathrm{DnaB}$ (in black) and the $H p \mathrm{DnaB} / H p \mathrm{DnaG}-\mathrm{CTD}$ complex (in green). The corresponding 1D traces are shown in Fig. S4. Black crosshairs mark assigned peaks that are observed in $H p D$ naB alone but not in the complex; purple crosses mark peaks that newly appear in the complex; and purple lines indicate the likely CSP shifts involved. Signal doubling is assigned to the asymmetric interaction with $H p D n a G$ (see text).

Additionally, appearing resonances of one Asn, one Asp and at least two Ile residues can be identified amino-acid type specifically. These signals are highlighted outside the grey boxes with blue arrows in Figs. 1 and S3. For these residues, no nearby disappearing signals could be identified.

\section{Discussion}


The interaction between DnaB and DnaG-CTD has been shown to involve mainly the Nterminal domain of the DnaB helicase. Indeed, its interaction with the DnaG primase has been revealed for B. stearothermophilus by X-ray crystallography (Bailey et al. 2007). For the $H$. pylori protein complex, we built a homology model of the $H p D n a B / H p D n a G-C T D$ protein complex based on the $H p$ DnaB (Bazin et al. 2015) and HpDnaG-CTD (Abdul Rehman et al. 2013) crystal structures and their alignment on the crystal structure of the $B s t \mathrm{DnaB} / B s t$ DnaGCTD complex (Bailey et al. 2007). To support the homology model, the sequence alignment of Bst vs. Hp for both DnaB and DnaG-CTD is shown in Figs. S5 and S6. The hexameric ring formed by the $H p D$ naB-NTDs is shown in grey in Fig. 3, with two monomers highlighted in green, and the $H p$ DnaG-CTD primase is shown in pink. Residues F66, I67, A88 and S89 from $H p D n a B$ for which disappearing signals were identified are highlighted in blue.

One primase is interacting with two monomers of the helicase, and it can be seen in Fig. 3A that for one of the monomers, the A88/S89 pair is located in this model right at the interaction interface, at the beginning of the loop contacting the HpDnaG-CTD. On the other monomer, the pair seems far from the interaction site. F66/I67 are, in one monomer, located at the interface between two $H p$ DnaB-NTD molecules, and on the other monomer they belong to a solvent-exposed helix. From the model, we predict that the shifts for residues A88 and S89 can be interpreted by conformational changes caused by the direct interaction with the $H p$ DnaG primase with the $H p$ DnaB helicase, whereas F66/I67 experience a perturbation due to changes at the $H p$ DnaB-NTD dimer interface upon binding. Still, the simple homology model does not account for all observations. It would predict that a signal with half the intensity would be left at the original position of the crosshairs in Fig. 2. Instead, this peak vanishes and a pair of new peaks (red crosses in Fig. 2) can be associated with the vanishing peak. Considering that the C-terminal hairpin of the DnaG primase has been shown to be flexible (Oakley et al. 2005; Syson et al. 2005), this behavior can however be predicted with a 
modified model where the hairpin adapts on binding to maximize the interaction interface. Such a model has been put forward using energetical arguments combined with mutational studies (Abdul Rehman et al. 2013). When considering this alternative conformation of HpDnaG-CTD (Abdul Rehman et al. 2013), as shown in Fig. 3B/C in dark red, the A88/S89 residues, as well as the F66/I67 of the other $H p D$ naB monomer, come close to the intermolecular interface, and can make contact to $H p$ DnaG-CTD. This would fully account for the shifting of the resonances to a pair of new positions, and our data thus strongly support an interaction in which the $H p$ DnaG-CTD hairpin is making contact, as proposed (Abdul Rehman et al. 2013), with the second monomer of $H p$ DnaB-NTD. This model also produces similar interaction interfaces as observed in the crystal structure of the BstDnaB/BstDnaGCTD complex (Bailey et al. 2007). There, the hairpin of BstDnaG-CTD indeed interacts with the second monomer of $B s t \mathrm{DnaB}$ as well. The conformation observed in the HpDnaG-CTD crystal structure might thus have been selected out of a variety of flexible conformations in solution for its ability to crystallize, but likely does not correspond to the one which is able to interact with $H p D$ DaB.

The signals which are observed only in the spectra of the $H p D$ naB/HpDnaG-CTD protein complex, and for which no neighboring missing peaks can be identified in the isolated $H p$ DnaB protein, must be located in regions in which only one monomer contacts $H p$ DnaG, and the other remains untouched. This would support the assignment of these signals to the loop containing residues I91-D92-N93-I94, which are shown in grey in Fig. 3C. This region remained unassigned in the isolated $H p D$ naB-NTD domain due to the absence of signal in the 3D spectra (Wiegand et al. 2015), which is indicative of it being dynamic in isolation. In the complex, this loop is in intimate contact with $H p$ DnaG-CTD for one $H p$ DnaB-Nter monomer, but not for the other, which yields a rational to the appearance of new resonances. Still, in 
order to confirm this hypothesis, more information has to be obtained to assess the identity of the appearing peaks.

\section{Conclusions}

Well-resolved NMR spectra can be obtained from a DnaB sediment alone and from a cosediment of the DnaB/DnaG protein complex. A comparison of the spectra allows us to identify residues that undergo large chemical-shift changes. In the context of a previously proposed model, we can interpret these changes and add experimental support to a so far theoretical model. In addition, the study illustrates opportunities and challenges when working with large protein assemblies where a full sequential assignment is presently extremely demanding. 

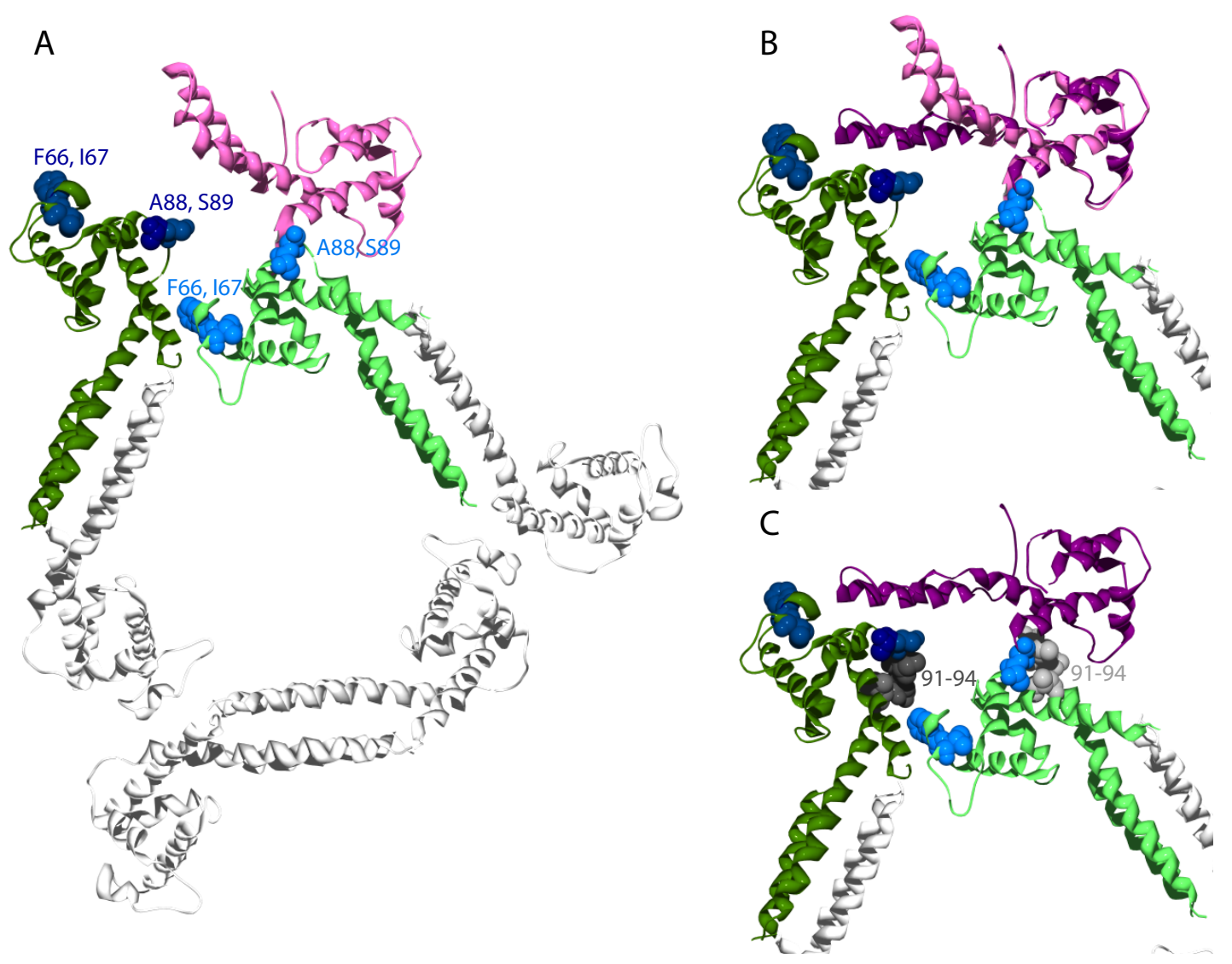

Figure 3: A: Homology model of the $H p D n a B / H p D n a G-C T D$ protein complex based on the $H p$ DnaB (Bazin et al. 2015) and HpDnaG-CTD (Abdul Rehman et al. 2013) crystal structures and their alignment on the crystal structure of the $B s t$ DnaB/BstDnaG-CTD complex (Bailey et al. 2007). Identified interacting residues F66, I67, A88 and S89 are highlighted in two different shades blue for the two non-equivalent monomers. B: An alternative conformation modeled for $H p D$ DaB-CTD built in order to satisfy interactions singled out in mutational studies (Abdul Rehman et al. 2013) is shown in violet. The globular part of the HpDnaG-CTD is aligned for the two proteins. C: Putative interacting residues 91-94 are shown in grey for the two monomers.

We have shown here how solid-state NMR of co-sedimented $H p$ DnaB helicase and $H p$ DnaGCTD primase can be used to reveal interaction interfaces in a large, multimeric and dynamic protein complex. Using the NMR derived restraints to guide homology-model building and 
taking into account the flexibility of the HpDnaG-CTD helical hairpin leads to a structural model which fully explains the spectral features identified, and in which the two proteins form an extended interface involving two $H p D n a B$ monomers. The identified interacting residues of $H p D n a B$ open the way to mutational studies to gain further insights into the helicase-primase interaction central in replication.

\section{Experimental Section}

\section{Protein expression and purification}

Uniformly ${ }^{13} \mathrm{C},{ }^{15} \mathrm{~N}$ enriched $H p$ DnaB was expressed from plasmid pACYC-duet1 in $E$. coli BL21 Star (DE3) cells (Invitrogen) using minimal M9-type media (Studier 2005) supplemented with uniformly ${ }^{13} \mathrm{C}$ enriched glucose $(2 \mathrm{~g} / \mathrm{L})$ and ${ }^{15} \mathrm{~N}$-labeled ammonium chloride $(2 \mathrm{~g} / \mathrm{L})$ as sole carbon and nitrogen sources. The protein was purified using affinity chromatography (5 mL HiTrap Heparin HP column from GE Healthcare) followed by anion exchange chromatography (5 mL HiTrap Q HP from GE Healthcare). Purified protein was in $10 \mathrm{mM}$ sodium phosphate buffer at $\mathrm{pH} 7.5$ containing $150 \mathrm{mM} \mathrm{NaCl}, 1 \mathrm{mM}$ DTT and $0.05 \%$ sodium azide. It was concentrated up to $30 \mathrm{mg} / \mathrm{mL}$ by centrifugation using Amicon Ultracel10K (Millipore).

The gene coding for HpDnaG-CTD (residues 415-559) was cloned into the plasmid pET151/D-TOPOR (Invitrogen). Unlabeled HpDnaG-CTD was expressed in E. coli BL21 Star (DE3) cells (Invitrogen) using LB medium. The protein was purified using affinity chromatography (5 mL HisTrap HP from GE Healthcare). After TEV-cleavage of the Nterminal His-tag, the protein was further purified by size-exclusion chromatography (Superdex 200 10/300GL column, GE Healthcare) in a buffer containing 50mM Tris- $\mathrm{HCl}$ at $\mathrm{pH} 8.0,200 \mathrm{mM} \mathrm{NaCl}, 1 \mathrm{mM}$ DTT and $5 \%$ glycerol. It was then concentrated up to $13 \mathrm{mg} / \mathrm{mL}$ by centrifugation using Amicon Ultracel-3K (Millipore). 
A mixture of $10 \mathrm{mg} H p$ DnaB and $10 \mathrm{mg} H p$ DnaG-CTD was concentrated up to $42 \mathrm{mg} / \mathrm{mL}$ total protein concentration using Amicon Ultracel-5K (Millipore) and sedimented by ultracentrifugation at $200000 \mathrm{~g}, 4{ }^{\circ} \mathrm{C}$ during 10 hours.

\section{NMR spectroscopy}

Solid-state NMR experiments were carried out on Bruker Biospin AVANCE III spectrometers operating at $800 \mathrm{MHz}$ and $850 \mathrm{MHz}{ }^{1} \mathrm{H}$ frequency using $3.2 \mathrm{~mm}$ tripleresonance $\left({ }^{1} \mathrm{H},{ }^{13} \mathrm{C},{ }^{15} \mathrm{~N}\right)$ E-free probes (Bruker Biospin). Sample temperature was determined using the chemical shift of supernatant water (Böckmann et al. 2009) and was adjusted to 278 K. Dipolar Assisted Rotational Resonance DARR (Takegoshi et al. 2001; Scholz et al. 2008) experiments were recorded with a mixing time of $20 \mathrm{~ms}$. Spectra were processed with Topspin using a shifted $\cos ^{2}$ function and analyzed using CcpNmr Analysis (Stevens et al. 2011). For full experimental parameters see Table S2.

\section{Model building}

$H p D n a B / H p D n a G-C T D$ models were built using the crystal structure of $H p D$ naB (pdb code 4ZC0). A hexamer of $H p D n a B$ was superimposed to the structure of $B s t$ DnaB/BstDnaG-CTD complex (pdb code 2R6A (Bailey et al. 2007)). Then three subunits of HpDnaG-CTD (pdb code 4EHS (Abdul Rehman et al. 2013)) were superimposed to the three BstDnaG-CTD subunits to generate a $H p D n a B_{6} / H p D n a G-C^{2} D_{3}$ complex. Minimal rearrangements were required to prevent small steric clashes between $H p$ DnaB and $H p$ DnaG-CTDs. The same method was used to generate the second $H p D n a B / H p D n a G-C T D$ model, but this time using three modified $H p$ DnaG-CTD models as described in (Abdul Rehman et al. 2013) and provided by Dr. Gourinath.

\section{Acknowledgments}


This work was supported by the French Agence Nationale de la Recherche (ANR-11-BSV8021-01, ANR-12-BS08- 0013-01, ANR-14-CE09-0024B), the ETH Zurich, the Swiss National Science Foundation (Grant 200020_159707 and 200020_146757). ABa and LT are supported by the CIBLE program 2011 from the Région Rhône-Alpes. We thank Professor Gourinath for providing us the coordinates of the HpDnaG-CTD model. We are grateful for support from TGIR-RMN-THC FR3050.

\section{References}

Abdul Rehman SA, Verma V, Mazumder M, et al (2013) Crystal structure and mode of helicase binding of the C-terminal domain of primase from Helicobacter pylori. $\mathrm{J}$ Bacteriol 195:2826-2838. doi: 10.1128/JB.00091-13

Bailey S, Eliason WK, Steitz TA (2007) Structure of Hexameric DnaB Helicase and Its Complex with a Domain of DnaG Primase. Science 318:459-463. doi: 10.1126/science. 1147353

Bazin A, Cherrier MV, Gutsche I, et al (2015) Structure and primase-mediated activation of a bacterial dodecameric replicative helicase. Nucleic Acids Res gkv792-13. doi: $10.1093 / \mathrm{nar} / \mathrm{gkv} 792$

Bertini I, Luchinat C, Parigi G, et al (2011) Solid-state NMR of proteins sedimented by ultracentrifugation. Proceedings of the National Academy of Sciences 108:10396-10399. doi: 10.1073/pnas.1103854108

Böckmann A, Gardiennet C, Verel R, et al (2009) Characterization of different water pools in solid-state NMR protein samples. J Biomol NMR 45:319-327. doi: 10.1007/s10858-0099374-3

Corn JE, Berger JM (2006) Regulation of bacterial priming and daughter strand synthesis through helicase-primase interactions. Nucleic Acids Res 34:4082-4088. doi: 10.1093/nar/gkl363

Gardiennet C, Schütz AK, Hunkeler A, et al (2012) A sedimented sample of a $59 \mathrm{kDa}$ dodecameric helicase yields high-resolution solid-state NMR spectra. Angew Chem Int Ed Engl 51:7855-7858. doi: 10.1002/anie.201200779

Kashav T, Nitharwal R, Abdulrehman SA, et al (2009) Three-dimensional structure of Nterminal domain of DnaB helicase and helicase-primase interactions in Helicobacter pylori. PLoS ONE 4:e7515. doi: 10.1371/journal.pone.0007515

Mainz A, Jehle S, van Rossum BJ, et al (2009) Large Protein Complexes with Extreme 
Rotational Correlation Times Investigated in Solution by Magic-Angle-Spinning NMR Spectroscopy. J Am Chem Soc 131:15968-15969. doi: 10.1021/ja904733v

Oakley AJ, Loscha KV, Schaeffer PM, et al (2005) Crystal and solution structures of the helicase-binding domain of Escherichia coli primase. J Biol Chem 280:11495-11504. doi: 10.1074/jbc.M412645200

Parsonnet J (1995) Bacterial infection as a cause of cancer. Environ Health Perspect 103 Suppl 8:263-268.

Scholz I, Huber M, Manolikas T, et al (2008) MIRROR recoupling and its application to spin diffusion under fast magic-angle spinning. Chemical Physics Letters 460:278-283. doi: 10.1016/j.cplett.2008.05.058

Stelter M, Gutsche I, Kapp U, et al (2012) Architecture of a dodecameric bacterial replicative helicase. Structure 20:554-564. doi: 10.1016/j.str.2012.01.020

Stevens TJ, Fogh RH, Boucher W, et al (2011) A software framework for analysing solidstate MAS NMR data. J Biomol NMR 51:437-447. doi: 10.1007/s10858-011-9569-2

Studier FW (2005) Protein production by auto-induction in high density shaking cultures. Protein Expression and Purification 41:207-234.

Su X-C, Schaeffer PM, Loscha KV, et al (2006) Monomeric solution structure of the helicasebinding domain of Escherichia coli DnaG primase. FEBS J 273:4997-5009. doi: 10.1111/j.1742-4658.2006.05495.x

Syson K, Thirlway J, Hounslow AM, et al (2005) Solution structure of the helicaseinteraction domain of the primase DnaG: a model for helicase activation. Structure/Folding and Design 13:609-616. doi: 10.1016/j.str.2005.01.022

Takegoshi K, Nakamura S, Terao T (2001) dipolar-assisted rotational resonance in magicangle spinning NMR. Chemical Physics Letters 344:631-637. doi: 10.1016/S00092614(01)00791-6

Wiegand T, Gardiennet C, Ravotti F, et al (2015) Solid-state NMR sequential assignments of the N-terminal domain of HpDnaB helicase. Biomol NMR Assign 1-11. doi: $10.1007 / \mathrm{s} 12104-015-9629-8$

Williamson MP (2013) Using chemical shift perturbation to characterise ligand binding. Progress in Nuclear Magnetic Resonance Spectroscopy 73:1-16. doi: 10.1016/j.pnmrs.2013.02.001 


\section{Supporting Information}

\section{Solid-state NMR chemical-shift perturbations indicate domain reorientation of the DnaG primase in the primosome of Helicobacter pylori}

Carole Gardiennet ${ }^{\mathrm{a} \uparrow}$, Thomas Wiegand ${ }^{\mathrm{b} \ddagger}$, Alexandre Bazin ${ }^{\mathrm{a}}$, Riccardo Cadalbert ${ }^{\mathrm{b}}$, Irina Gutsche $^{\mathrm{c}}$, Britta Kunert ${ }^{\mathrm{a}}$, Denis Lacabanne ${ }^{\mathrm{a}}$, Laurent Terradot ${ }^{\mathrm{a}^{*}}$, Beat H. Meier ${ }^{\mathrm{b}^{*}}$, and Anja

\section{Böckmann $^{\mathrm{a}^{*}}$}

${ }^{a}$ Molecular Microbiology and Structural Biochemistry, Labex Ecofect, UMR 5086 CNRS, Université de Lyon 1, 7 passage du Vercors, 69007 Lyon, France

${ }^{b}$ Physical Chemistry, ETH Zurich, Vladimir-Prelog-Weg 2, 8093 Zurich (Switzerland)

${ }^{c}$ Unit of Virus Host-Cell Interactions, UJF-EMBL-CNRS, UMI3265, 38042 Grenoble Cedex 9 , France

${ }^{\dagger}$ present address : CNRS, Université de Lorraine, CRM2, UMR 7036, F-54506 Vandoeuvrelès-Nancy, France

${ }^{a}$ Institut de Biologie et Chemie des Protéines, Bases Moléculaires et Structurales des Systèmes Infectieux, Labex Ecofect, UMR 5086 CNRS, Université de Lyon, 7 passage du Vercors, 69007 Lyon, France

${ }^{b}$ Physical Chemistry, ETH Zurich, Vladimir-Prelog-Weg 2, 8093 Zurich (Switzerland)

${ }^{c}$ Unit of Virus Host-Cell Interactions, UJF-EMBL-CNRS, UMI3265, 38042 Grenoble Cedex 9, France

${ }^{\dagger}$ present address : CNRS, Université de Lorraine, CRM2, UMR 7036, F-54506 Vandoeuvrelès-Nancy, France 


\section{A}

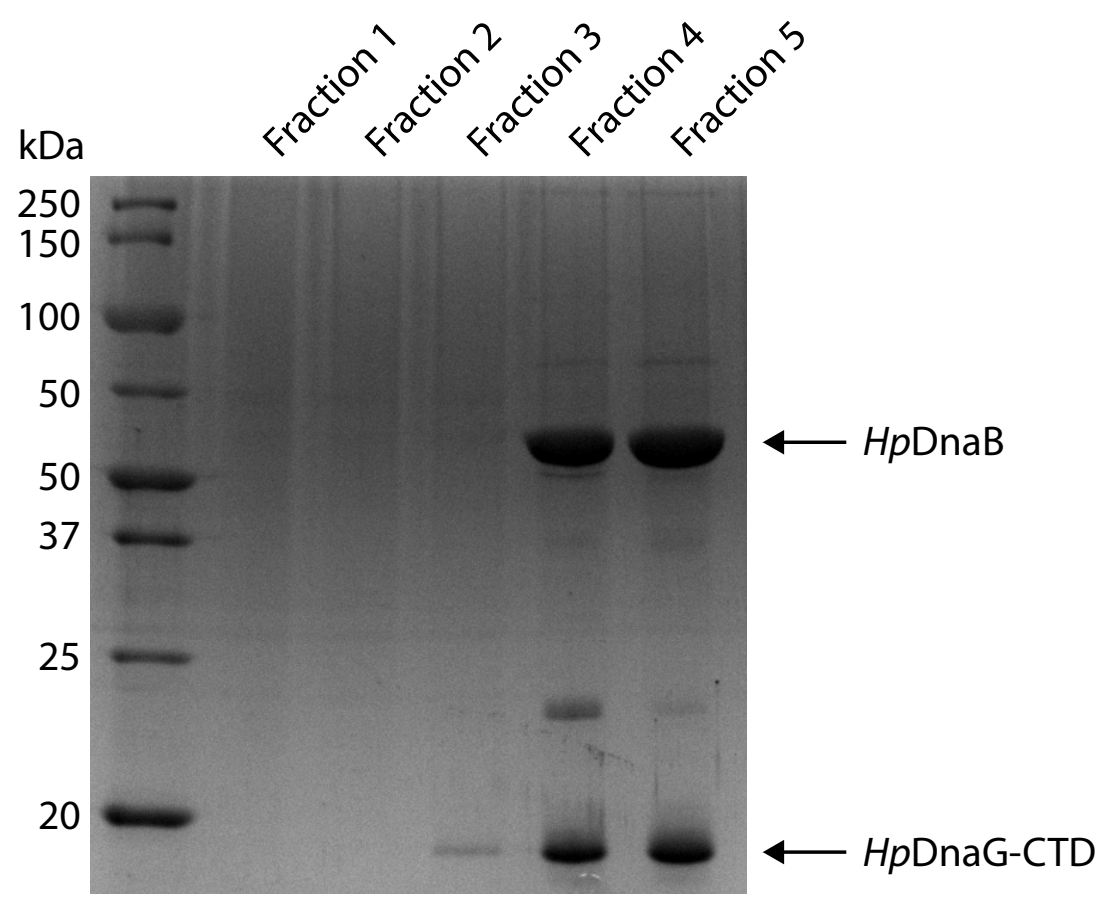

B

\begin{tabular}{llllll} 
& F1 & F2 & F3 & F4 & F5 \\
\hline A280 & 0.47 & 0.54 & 0.83 & 5.54 & 8.96 \\
{$[\mathrm{C}](\mathrm{mg} / \mathrm{mL})$} & 1.18 & 1.35 & 2.08 & 13.84 & 22.41 \\
Total amount of protein $(\mathrm{mg})$ & 0.01 & 0.01 & 0.02 & 0.14 & 0.45
\end{tabular}

Figure S1: A: Coomassie-blue stained SDS-PAGE analysis of an analytical run of $H p \mathrm{DnaB} / \mathrm{H} p \mathrm{DnaG}-\mathrm{CTD}$ co-sedimentation; the lanes correspond to the different fractions in the tube after ultracentrifugation at $200000 \mathrm{~g}$ overnight going from 1(top) to 5 (bottom of the tube). Fractions 1-4 were each $10 \mu \mathrm{l}$ and the pellet (fraction 5) was resuspended in $20 \mu \mathrm{l}$ Tris$\mathrm{HCl}$ buffer at $\mathrm{pH} 8.0$ for analysis. $5 \mu$ of each fraction were loaded on the Coomassie-blue stained gel. The initial total protein concentration of the sample $(H p \mathrm{DnaB}+H p \mathrm{DnaG}-$ CTD) was $25 \mathrm{mg} / \mathrm{ml}$. In B is shown the absorption measured at $280 \mathrm{~nm}$ of the different fractions, and the resulting concentrations. The total amount of protein in each fraction is calculated taking into account the total volume of each fraction. The $0.45 \mathrm{mg}$ found in fraction 5 corresponds to $70 \%$ of the total sample. In order to achieve even higher efficiency, 
the protein solution used for sedimentation of the NMR sample was concentrated to $42 \mathrm{mg} / \mathrm{ml}$ (see Methods section in main text). Some DnaG-CTD is found in fraction 3 as it was added in excess to the theoretical 2:1 ratio of $H p$ DnaB: $H p D$ DaG-CTD. 


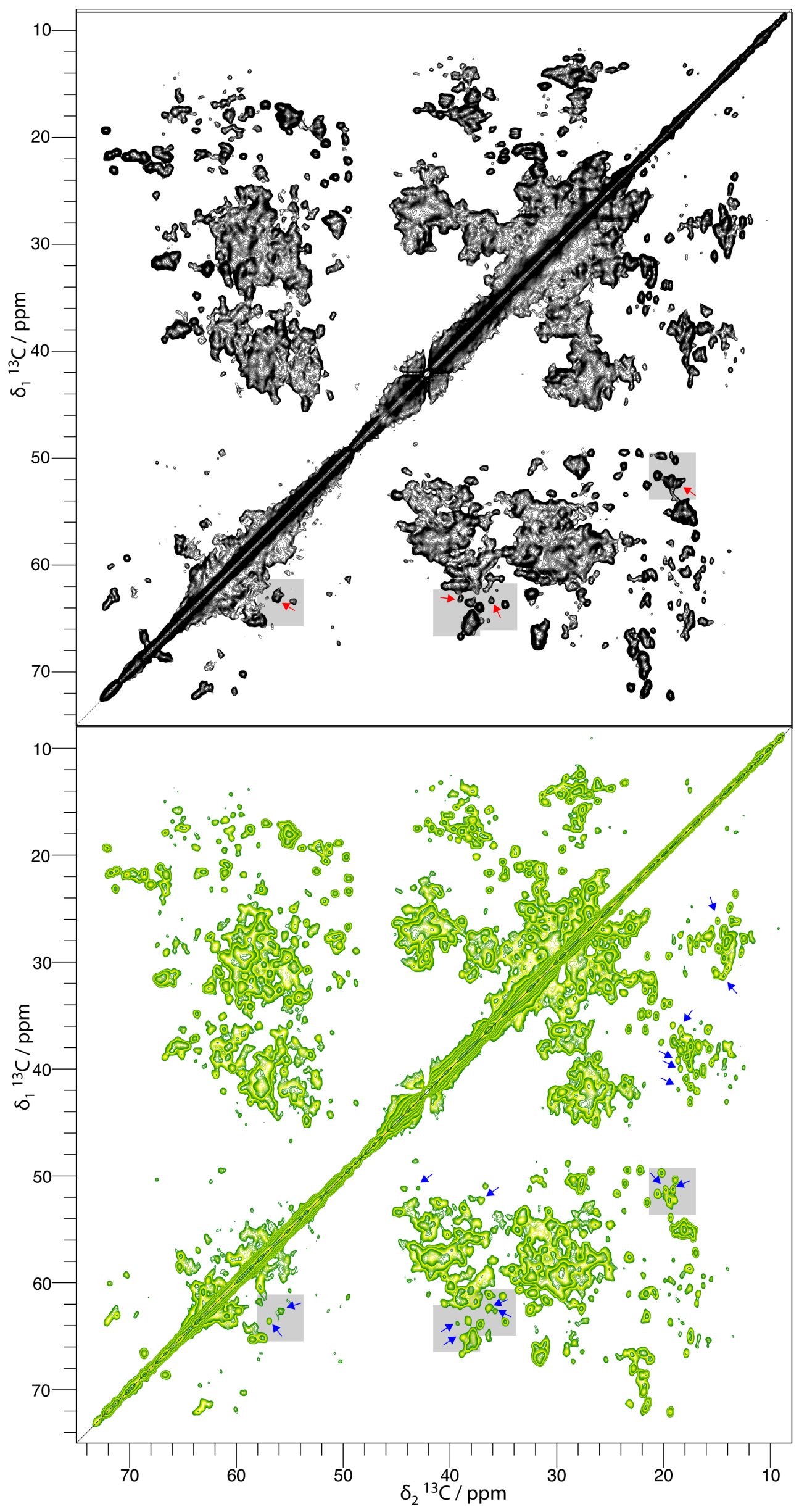


Figure S2: Aliphatic region of DARR spectra of $H p D n a B$ (black, top) and $H p \mathrm{DnaB} / \mathrm{HpDnaG-CTD}$ (green, bottom) recorded with $20 \mathrm{~ms}$ mixing time. Red arrows indicate signals only observed in the $\mathrm{HpDnaB}$, and blue arrows indicate signals only observed in the protein complex.

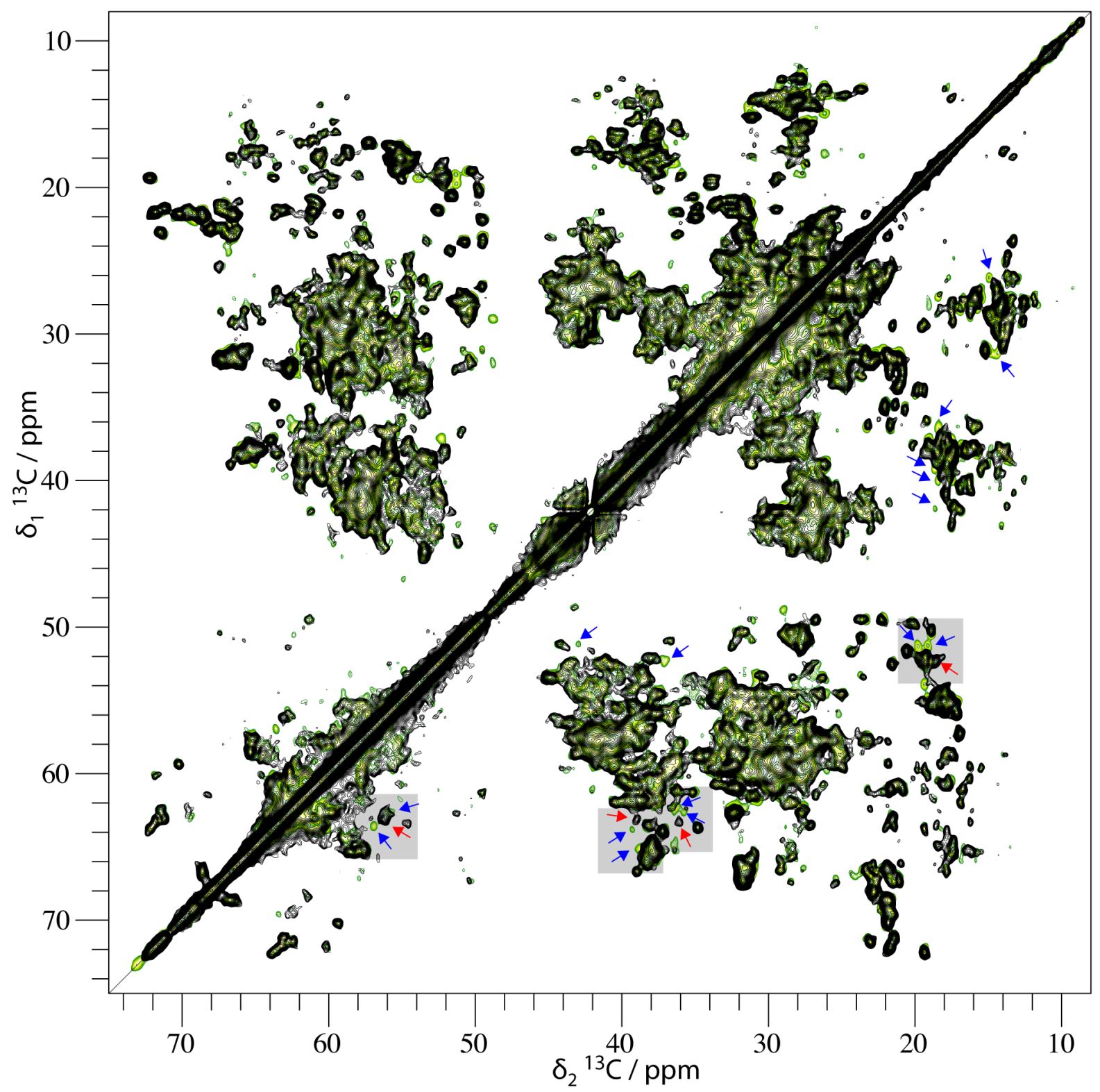

Figure S3: Overlay of the full aliphatic region of DARR spectra recorded with $20 \mathrm{~ms}$ mixing time. In green the spectrum of the $H p D$ DaB:HpDnaG-CTD complex, and in black the spectrum of the HpDnaB protein. Red arrows indicate signals only observed in the HpDnaB, and blue arrows indicate signals only observed in the protein complex. 


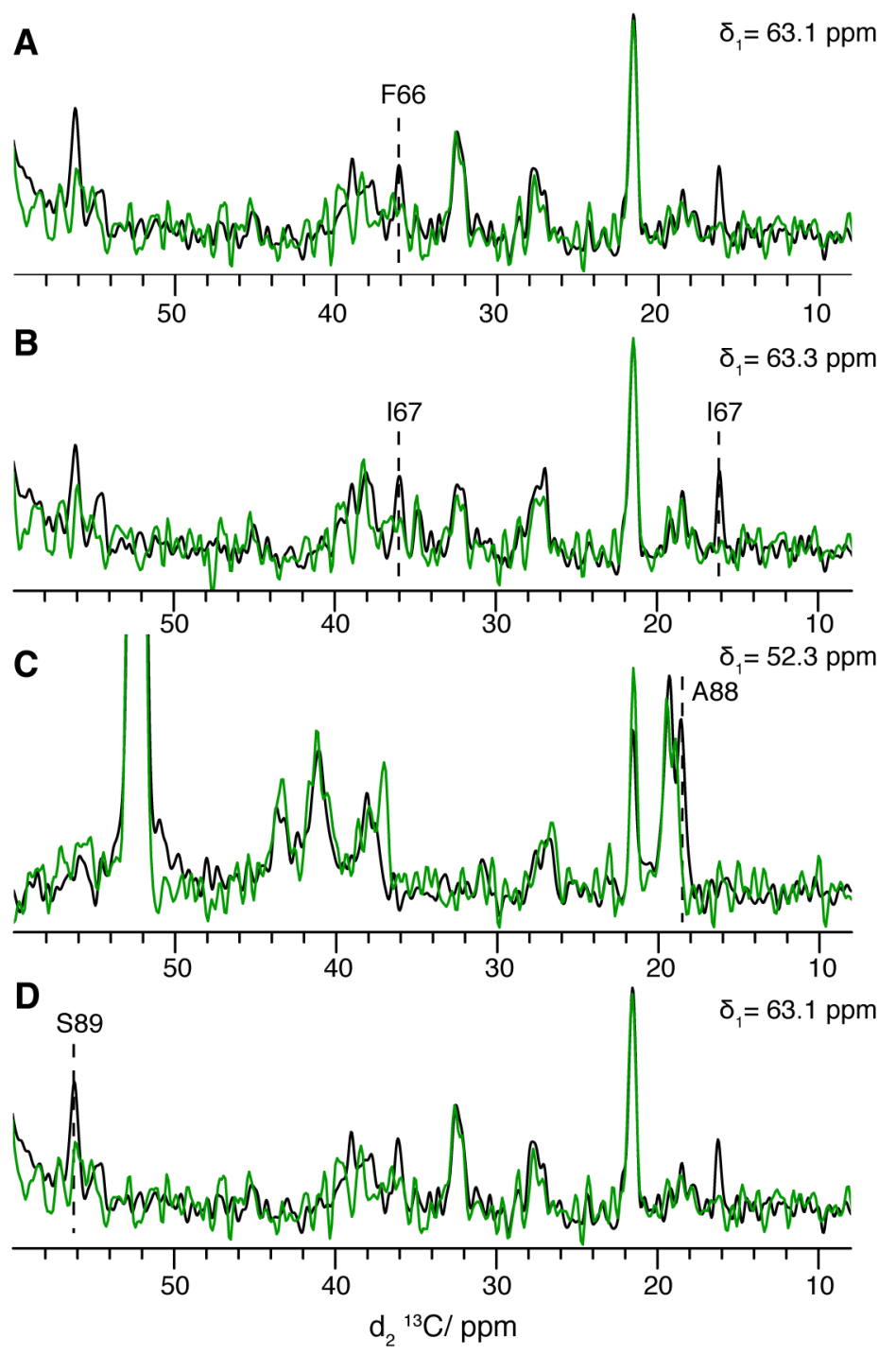

Figure S4: 1D traces corresponding to signals of extracts shown in Figure 2. 


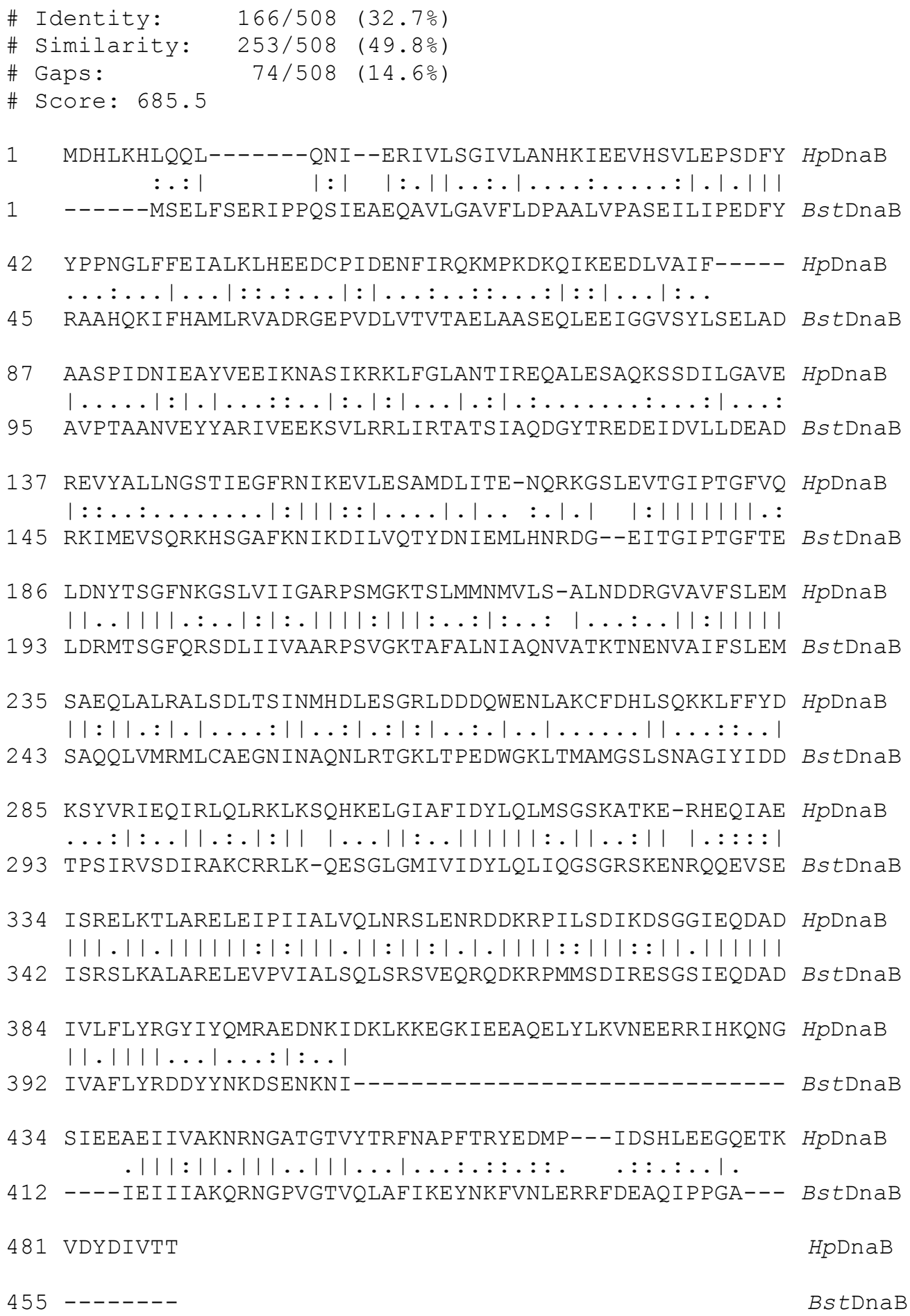

Figure S5: Sequence alignment of $\underline{H p D n a B}$ and $B s t$ DnaB using EMBOSS (version 6.6.0) (Rice et al. 2000). Legend: identity (|), similarity with a small positive score (.) and similarity with a score larger $1.0(:)$. 


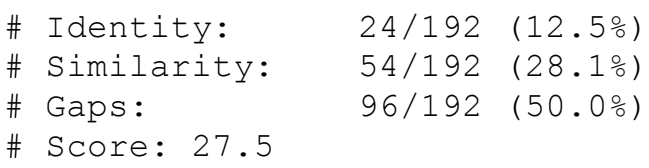

Figure S6: Sequence alignment of $H p D n a G(C T D)$ and BstDnaG(CTD) using EMBOSS (version 6.6.0) (Rice et al. 2000). Legend: identity (|), similarity with a small positive score (.) and similarity with a score larger $1.0(:)$.

\section{References}

Rice P, Longden I, Bleasby A (2000) EMBOSS: the European molecular biology open software suite. 
Table S1: Chemical shift differences between the signals disappearing in the $H p D n a B$ sediment and their nearby appearing signals in the $H p D n a B / H p D n a G-C T D$ co-sediment as shown in Figure 2.

\begin{tabular}{lcccc} 
Residue & Atom Name & $\delta$ DnaB $(\mathrm{ppm})$ & $\delta$ DnaB/DnaG $(\mathrm{ppm})$ & $\Delta \delta(\mathrm{ppm})$ \\
\hline F66 & $\mathrm{C} \alpha$ & 63.17 & 63.81 & 0.63 \\
& & & 65.12 & 1.95 \\
F66 & $\mathrm{C} \beta$ & 39.00 & 39.30 & 0.30 \\
& & & 38.74 & 1.98 \\
$\mathrm{I} 67$ & $\mathrm{C} \alpha$ & 63.24 & 62.63 & -0.61 \\
& & & 62.36 & -0.88 \\
$\mathrm{I} 67$ & $\mathrm{C} \beta$ & 36.12 & 35.72 & -0.40 \\
& & & 36.39 & 0.27 \\
A88 & $\mathrm{C} \alpha$ & 52.24 & 51.27 & -0.97 \\
& & & 51.28 & -0.96 \\
A88 & $\mathrm{C} \beta$ & \multirow{2}{*}{18.57} & 19.14 & 0.57 \\
& & & 19.80 & 1.23 \\
S89 & $\mathrm{C} \alpha$ & 56.17 & 56.91 & 0.74 \\
& & & 55.84 & -0.33 \\
S89 & $\mathrm{C} \beta$ & 63.16 & 63.61 & 0.45 \\
& & & 62.66 & -0.50
\end{tabular}


Table S2 : Overview about experimental parameters of the performed solid-state NMR experiments.

\begin{tabular}{|c|c|c|c|c|}
\hline Experiment/ Sample & $\begin{array}{l}\text { DARR/ } \\
\text { NTD } \\
\text { HpDnaB }\end{array}$ & $\begin{array}{l}\text { DARR/ } \\
\text { CTD } \\
\text { HpDnaB }\end{array}$ & $\begin{array}{l}\text { DARR/ } \\
H p \text { DnaB }\end{array}$ & $\begin{array}{l}\text { DARR/ } \\
\text { HpDnaB:HpDna } \\
\text { G-CTD }\end{array}$ \\
\hline MAS frequency/ kHz & 17.0 & 17.5 & 17.0 & 17.5 \\
\hline Field/ T & 20.0 & 18.8 & 20.0 & 18.8 \\
\hline Transfer I & $\mathrm{HC}-\mathrm{CP}$ & $\mathrm{HC}-\mathrm{CP}$ & $\mathrm{HC}-\mathrm{CP}$ & $\mathrm{HC}-\mathrm{CP}$ \\
\hline${ }^{1} \mathbf{H}$ field/ $\mathbf{k H z}$ & 59.4 & 65.0 & 58.6 & 64.0 \\
\hline $\mathrm{X}$ field/ $\mathbf{k H z}$ & 40.9 & 50.0 & 41.6 & 50.0 \\
\hline Shape & Tangent ${ }^{1} \mathrm{H}$ & Tangent ${ }^{1} \mathrm{H}$ & Tangent ${ }^{1} \mathrm{H}$ & Tangent ${ }^{1} \mathrm{H}$ \\
\hline${ }^{13} \mathrm{C}$ carrier/ ppm & 95 & 59 & 103 & 55 \\
\hline Time/ ms & 0.5 & 0.7 & 0.5 & 0.7 \\
\hline Transfer II & DARR & DARR & DARR & DARR \\
\hline${ }^{1} \mathrm{H}$ field/ $\mathrm{kHz}$ & 17.0 & 17.5 & 17.0 & 17.5 \\
\hline Carrier/ ppm & 95 & 59 & 103 & 55 \\
\hline Time/ ms & 20 & 20 & 20 & 20 \\
\hline t1 increments & 2000 & 1876 & 2560 & 2000 \\
\hline Sweep width $\left(t_{1}\right) / \mathbf{k H z}$ & 100 & 94 & 100 & 94 \\
\hline Acquisition time $\left(t_{1}\right) / m s$ & 10 & 10 & 13 & 11 \\
\hline t2 increments & 2988 & 2802 & 3072 & 2988 \\
\hline Sweep width $\left(t_{2}\right) / \mathbf{k H z}$ & 100 & 94 & 100 & 94 \\
\hline Acquisition time $\left(t_{2}\right) / m s$ & 14.9 & 14.9 & 15.4 & 16.0 \\
\hline $\begin{array}{l}{ }^{1} \mathrm{H} \text { Spinal64 decoupling } \\
\text { power/ kHz }\end{array}$ & 89 & 90 & 90 & 90 \\
\hline Interscan delay/s & 2.1 & 2.1 & 2.5 & 2.2 \\
\hline Number of scans & 12 & 12 & 16 & 36 \\
\hline Measurement time/ $h$ & 14 & 13 & 28 & 45 \\
\hline
\end{tabular}

